\title{
Genetics of serum resistin: a paradigm of population-specific regulation?
}

\author{
C. Menzaghi $\cdot$ V. Trischitta
}

Received: 15 September 2009 / Accepted: 5 October 2009 /Published online: 1 November 2009

(C) Springer-Verlag 2009

Keywords Insulin resistance - Population-specific effect . Resistin circulating levels $\cdot$ RETN

Adipocytes release a number of peptide hormones, collectively known as adipokines, which are essential in regulation of intermediate metabolism, and which may contribute to the pathogenesis of insulin resistance and related disorders [1].

Among these is resistin, a $12.5 \mathrm{kDa}$ cysteine-rich protein that is secreted also by macrophages [2-4]. Several pieces of evidence suggest that this molecule antagonises insulin action and fosters inflammation [2-4]. In humans, most cross-sectional studies have reported that elevated resistin levels are associated with insulin resistance [5-7] and type 2 diabetes [8]. In most [9-11], although not all [12], prospective studies, resistin turned out to be a predictor of type 2 diabetes and cardiovascular disease. Taken together, these findings highlight the role of serum resistin as an

C. Menzaghi $(\triangle) \cdot$ V. Trischitta

Research Unit of Diabetes and Endocrine Diseases,

IRCCS 'Casa Sollievo della Sofferenza', Viale Padre Pio,

71013 San Giovanni Rotondo (FG), Italy

e-mail: c.menzaghi@operapadrepio.it

V. Trischitta

Department of Medical Pathophysiology, 'Sapienza' University,

Rome, Italy

V. Trischitta

IRCCS 'Casa Sollievo della Sofferenza'-Mendel Institute,

Rome, Italy emerging pathogenic factor and a potential therapeutic target for insulin resistance-related disorders. This latter possibility is reinforced by the observation that resistin expression in human adipose tissue is inhibited by thiazolidinediones [13], insulin-sensitising molecules that are used as oral hypoglycaemic agents in the treatment of patients with type 2 diabetes.

The mechanisms regulating resistin expression, secretion and circulating levels are still poorly understood. Recent data clearly indicate that resistin serum levels are under genetic control $[6,14]$. Several single-nucleotide polymorphisms (SNPs) in the RETN gene coding for resistin, have been associated with serum resistin levels and insulin resistance traits [6, 8, 14-18].

Among others, rs1862513 at position g. -420 in the RETN $5^{\prime}$ flanking region has attracted much attention. This SNP has been associated with high resistin levels $[8,16]$, insulin resistance [17], obesity $[15,17,18]$ and type 2 diabetes $[8,19]$. A recent meta-analysis, including more than 3,500 individuals, has shown that those homozygous for the $\mathrm{G}$ allele at rs 1862513 have a $30 \%$ increase in the odds of being affected by type 2 diabetes [8]. Along the same lines, the $G$ allele was predictive of glycaemic deterioration in a Chinese population followed for 5 years [20]. These associations are supported by functional data demonstrating a stronger activation of the RETN promoter by $\mathrm{Sp} 1$ and $\mathrm{Sp} 3$ transcription factors in the presence of the $\mathrm{G}$ allele [8]. In agreement with these data, the same allele has been reported to be associated with higher abdominalfat resistin mRNA levels [21]. Thus, so far, so good for SNP rs1862513.

Recently, a fine-mapping analysis of the RETN gene has been carried out in a sample of more than 2,500 individuals of European ancestry [14]. The authors found that alleles at 
rs1477341, rs4804765, rs1423096 and rs10401670 in the gene $3^{\prime}$ UTR were associated with circulating resistin levels; the latter was associated also with fasting glucose and, once SNP-by-BMI interaction was taken into account, with incidence of type 2 diabetes. Quite surprisingly, SNP rs1862513 was not associated with resistin levels [14]. When all published data on more than 5,000 individuals were meta-analysed, a significant association was observed between this SNP and resistin levels; however, it was mainly driven by data obtained in Asian populations, thus suggesting some heterogeneity caused by ethnic-specific genetic effects [14].

In this issue of Diabetologia, Asano et al. [22] have analysed the relationships between serum resistin levels, metabolic traits and RETN variants in a cross-sectional study, carried out in a large cohort comprising more than 3,000 aged Japanese. Like Hivert et al. [14] they used a fine-mapping approach, although limited to the RETN gene. With a straightforward study design and an accurate methodology, they found that two SNPs (i.e. rs34861192 in the promoter and rs3745368 in the 3' UTR region) were significantly and independently associated with serum resistin, explaining a very large proportion $(\sim 40 \%)$ of its variability.

Of note, while resistin levels were associated with HDLcholesterol, triacylglycerol and obesity, no significant associations were observed between rs34861192 and rs3745368 and these metabolic traits, or the prevalence of type 2 diabetes. Finally, a significant association was observed also for the promoter SNP rs1862513, which, however, was no longer significant in a multiple SNP model, being mostly driven by rs34861192.

Asano et al. wanted to dissect cause-effect relationships among the observed associations by using Mendelian randomisation, an approach to robustly identify causal correlations [23]. However, when a genetic variant is associated with an intermediate phenotype, but not with disease outcome (i.e. resistin levels and metabolic traits, respectively, in this study), the usefulness of such an approach is highly questionable and results remain inconclusive [24].

The study by Asano et al. extends previous investigations in several important aspects. First, it further confirms the association between variability at the RETN locus and circulating resistin levels in a Japanese population.

Second, it shows that this association depends on polymorphisms in both the promoter and the $3^{\prime}$ UTR unique to this sample and different from those previously reported in other Asian [8, 16] and European [14] samples. Taken together, these and previous data reinforce the possibility of a population-specific genetic effect [14]. Variability of the RETN gene across different samples, including discrepancies in haplotype structure, allele frequencies (with rs3745368 being very rare in Europeans
[14]) and the presence of monomorphism (with Europeans carrying only the GG genotype at rs34861192 [22]), might explain such a specific effect. Differences across populations in environmental exposures (e.g. different dietary habits) and/or in genetic background might have also played a role. Inter- and intra-ethnic different genetic associations have been recently reported for type 2 diabetes, with the effect of PPARG P12A SNP, an established genetic marker of this disease, being stronger in Asians than in Europeans and among the latter being easily appreciable in northern populations and virtually absent in the Mediterranean area [25]. Placing this issue in a broader perspective, present and previous findings strongly suggest that population-specific genetic effects may occur and, therefore, should be always considered when analysing and interpreting data on the genetics of complex traits.

Third, in this Japanese cohort, the combination of only two RETN variants may modulate a proportion of resistin levels variability that is more than an order of magnitude higher than that previously reported for resistin itself [6, 14], and definitely uncommon in the dominion of quantitative traits, including other adipokines [26, 27]. In the present understanding of the role of common genetic variants that are likely to exert minor effects, this result is totally unexpected and definitively deserves confirmation in further studies.

The genetics of adipokines is still an open and constantly growing area of interest in understanding the pathophysiology of insulin resistance traits. The paper by Asano et al. [22] has made some progress in dissecting the contribution of the RETN locus to resistin circulating levels; further studies are still needed to unravel whether this contribution is really different in different populations. To this purpose, a multicentre collaborative effort, able to recruit and analyse several large samples of different ethnicity, is desirable. In addition, functional studies are necessary to investigate whether the variants so far described, and possibly others yet to be identified, influence RETN transcriptional activity and mRNA levels and/or resistin protein synthesis and production in appropriate tissues. A final and very challenging aim is to examine the genome-wide SNPs and expression datasets in a variety of populations. This may lead to the identification of additional genes able to explain the still large proportion of serum resistin genetic variance that is not accounted for by polymorphisms at the RETN locus.

Acknowledgements Supported by Accordo Programma Quadro in Materia di Ricerca Scientifica nella Regione Puglia-PST 2006 and Italian Ministry of Health grants Ricerca Corrente 2005-2009 (C. Menzaghi).

Duality of interest The authors declare that there is no duality of interest associated with this manuscript. 


\section{References}

1. Scherer PE (2006) Adipose tissue: from lipid storage compartment to endocrine organ. Diabetes 55:1537-1545

2. Steppan CM, Bailey ST, Bhat S et al (2001) The hormone resistin links obesity to diabetes. Nature 409:307-312

3. Steppan CM, Lazar MA (2004) The current biology of resistin. J Intern Med 255:439-447

4. Reilly MP, Lehrke M, Wolfe ML, Rohatgi A, Lazar MA, Rader DJ (2005) Resistin is an inflammatory marker of atherosclerosis in humans. Circulation 111:932-939

5. Heilbronn LK, Rood J, Janderova L et al (2004) Relationship between serum resistin concentrations and insulin resistance in nonobese, obese, and obese diabetic subjects. J Clin Endocrinol Metab 89:1844-1848

6. Menzaghi C, Coco A, Salvemini L et al (2006) Heritability of serum resistin and its genetic correlation with insulin resistancerelated features in nondiabetic Caucasians. J Clin Endocrinol Metab 91:2792-2795

7. Hivert MF, Sullivan LM, Fox CS et al (2008) Associations of adiponectin, resistin, and tumor necrosis factor-alpha with insulin resistance. J Clin Endocrinol Metab 93:3165-3172

8. Osawa H, Yamada K, Onuma H et al (2004) The G/G genotype of a resistin single-nucleotide polymorphism at -420 increases type 2 diabetes mellitus susceptibility by inducing promoter activity through specific binding of Sp1/3. Am J Hum Genet 75:678-686

9. Chen BH, Song Y, Ding EL et al (2009) Circulating levels of resistin and risk of type 2 diabetes in men and women: results from two prospective cohorts. Diabetes Care 32:329-334

10. Weikert C, Westphal S, Berger K et al (2008) Plasma resistin levels and risk of myocardial infarction and ischemic stroke. J Clin Endocrinol Metab 93:2647-2653

11. Frankel DS, Vasan RS, D'Agostino RB Sr et al (2009) Resistin, adiponectin, and risk of heart failure: the Framingham Offspring Study. J Am Coll Cardiol 53:754-762

12. Heidemann C, Sun Q, van Dam RM et al (2008) Total and highmolecular-weight adiponectin and resistin in relation to the risk for type 2 diabetes in women. Ann Intern Med 149:307-316

13. Kolak M, Yki-Jarvinen H, Kannisto K et al (2007) Effects of chronic rosiglitazone therapy on gene expression in human adipose tissue in vivo in patients with type 2 diabetes. J Clin Endocrinol Metab 92:720-724

14. Hivert MF, Manning AK, McAteer JB et al (2009) Association of variants in RETN with plasma resistin levels and diabetes-related traits in the Framingham Offspring Study. Diabetes 58:750-756
15. Engert JC, Vohl MC, Williams SM et al (2002) 5' flanking variants of resistin are associated with obesity. Diabetes 51:1629 1634

16. Cho YM, Youn BS, Chung SS et al (2004) Common genetic polymorphisms in the promoter of resistin gene are major determinants of plasma resistin concentrations in humans. Diabetologia 47:559-565

17. Conneely KN, Silander K, Scott LJ et al (2004) Variation in the resistin gene is associated with obesity and insulin-related phenotypes in Finnish subjects. Diabetologia 47:1782-1788

18. Mattevi VS, Zembrzuski VM, Hutz MH (2004) A resistin gene polymorphism is associated with body mass index in women. Hum Genet 115:208-212

19. Ochi M, Osawa H, Hirota $Y$ et al (2007) Frequency of the $G / G$ genotype of resistin single nucleotide polymorphism at -420 appears to be increased in younger-onset type 2 diabetes. Diabetes 56:2834-2838

20. Xu JY, Sham PC, Xu A et al (2007) Resistin gene polymorphisms and progression of glycaemia in southern Chinese: a 5-year prospective study. Clin Endocrinol (Oxf) 66:211-217

21. Smith SR, Bai F, Charbonneau C, Janderova L, Argyropoulos G (2003) A promoter genotype and oxidative stress potentially link resistin to human insulin resistance. Diabetes 52:1611-1618

22. Asano H, Izawa $H$, Nagata $K$ et al (2009) Plasma resistin concentration determined by common variants in the resistin gene and associated with metabolic traits in an aged Japanese population. Diabetologia. doi:10.1007/s00125-009-1517-2

23. Sheehan NA, Didelez V, Burton PR, Tobin MD (2008) Mendelian randomisation and causal inference in observational epidemiology. PLoS Med 5:e177

24. Nitsch D, Molokhia M, Smeeth L, DeStavola BL, Whittaker JC, Leon DA (2006) Limits to causal inference based on Mendelian randomization: a comparison with randomized controlled trials. Am J Epidemiol 163:397-403

25. Ludovico O, Pellegrini F, Di Paola R et al (2007) Heterogeneous effect of peroxisome proliferator-activated receptor gamma2 Ala12 variant on type 2 diabetes risk. Obesity (Silver Spring) 15:1076-1081

26. Menzaghi C, Trischitta V, Doria A (2007) Genetic influences of adiponectin on insulin resistance, type 2 diabetes, and cardiovascular disease. Diabetes 56:1198-1209

27. Hivert MF, Manning AK, McAteer JB et al (2008) Common variants in the adiponectin gene (ADIPOQ) associated with plasma adiponectin levels, type 2 diabetes, and diabetes-related quantitative traits: the Framingham Offspring Study. Diabetes 57:3353-3359 